\title{
DETECTION DE L'HYDROLYSE PARTIELLE DU LACTOSE DANS UN ECHANTILLON DE LAIT PAR CHROMATOGRAPHIE EN COUCHE MINCE
}

\author{
par \\ B. DEMANGE et R. FRENTZ
}

La recherche du mouillage d'un lait implique généralement le calcul de sa constante moléculaire simplifiée (ou CMS) qui fait entrer en jeu sa teneur en chlorures et en lactose. Or un dosage correct de ce sucre par la méthode réductimétrique ne peut se faire que si son hydrolyse en glucose et galactose n'introduit pas une erreur supérieure à celle officiellement admise pour la méthode utilisée, soit $\pm 0,3 \mathrm{~g} / 1$. Dans le cas contraire, il devient indispensable de provoquer l'hydrolyse totale du lactose avant de passer à l'analyse. Mme I. Saenz Lascano Ruiz [1] a montré qu'un gramme de lactose. hydraté par litre, totalement hydrolysé et donc dosé sous forme de glucose-galactose, équivaut, du point de vue analytique, à $1,451 \mathrm{~g}$ du diholoside dosé sous sa forme intacte. L'erreur de $0,45 \mathrm{~g} / 1$ commise dans ces conditions est supérieure à celle qui est tolérée et on peut en déduire que pour faire un dosage du lactose conforme aux exigences de la législation, il est indispensable que, par litre, la quantité de lactose hydrolysé soit inférieure à $0,5 \mathrm{~g}$, l'erreur introduite n'étant alors que de $0,225 \mathrm{~g}$.

Dans un lait absolument frais le lactose n'est pratiquement pas encore dégradé et son dosage ne pose pas de problème particulier. Cela n'est plus le cas pour un lait plus ancien, traité ou conservé. Il devient alors indispensable, avant de doser le lactose, de s'assurer que son hydrolyse n'a pas dépassé le taux de $0,5 \mathrm{~g} / 1$. Pour ce faire, Mme I. Saenz Lascano Ruiz [1] a élaboré une technique chromatographique sur papier, semi-quantitative, qui permet d'apprécier si, dans un échantillon donné, le seuil d'hydrolyse de $0,5 \mathrm{~g}$ de lactose par litre a été dépassé. Cette méthode, parfaitement valable quant à son principe et à ses résultats, présente néanmoins l'inconvénient d'être relativement longue puisque la lecture ne peut être faite qu'après un délai de 40 heures au moins, ce qui reporte assez loin la deuxième phase d'une hydrolyse éventuelle.

Cherchant à mettre à profit les lavantages qu'apporte à ce point de vue la chromatographie en couche mince de $E$. Stahl [2], nous nous sommes efforcés d'adapter cette dernière au problème posé et de dégager le mode opératoire le plus favorable. 


\section{I - Principe}

Après une défécation courante au ferrocyanure de $\mathbf{K}$ et à l'acétate de zinc pour les laits normaux ou formolés et, en plus, au chlorure de baryum pour les laits bichromatés, on réalise une chromatographie en couche mince de gel de silice du filtrat obtenu, parallèlement à des solutions-étalons de sucres contenant respectivement :

$-0,250 \mathrm{~g}$ de glucose $+0,250 \mathrm{~g}$ de galactose (équivalents à $0,5 \mathrm{~g}$ de lactose hydrolysé) $+49,5 \mathrm{~g}$ de lactose par litre ;

$-0,500 \mathrm{~g}$ de glucose $+0,500 \mathrm{~g}$ de galactose (équivalents à $1 \mathrm{~g}$ de lactose hydrolysé) $+49 \mathrm{~g}$ de lactose par litre.

Après une migration de 20 à 25 minutes et une révélation au phtalate d'aniline, on compare en lumière normale ou ultra-violette les taches de l'échantillon à celles des hexoses-étalons, ce qui permet de conclure si le lait étudié contient plus ou moins de $0,5 \mathrm{~g}$ de lactose hydrolysé par litre, donc s'il doit être oui ou non soumis à une hydrolyse totale ultérieure.

\section{II. - Mode opératoire}

\section{A. - Préparation des solutions à chromatographier.}

1) Filtrat lactique.

a) Matériel et réactifs :

- Un ballon jaugé de $200 \mathrm{ml}$;

- Solution aqueuse de ferrocyanure de potassium Fe $(\mathrm{CN})_{6}$ $\mathrm{K}_{4}, 3 \mathrm{H}_{2} \mathrm{O}$ à 15 p. 100 (solution A);

- Solution aqueuse d'acétate de zinc $\left(\mathrm{CH}_{3} \mathrm{COO}\right)_{2} \mathrm{Zn}, 2 \mathrm{H}_{2} \mathrm{O}$ à 30 p. 100 (solution B); tion $\mathrm{C})$ ?

- Phénolphtaléine en solution alcoolique à 0,5 p. 100 (solu-

- Liqueur alcaline normale de potasse $\mathrm{KOH}$ (solution D);

- Solution de chlorure de baryum à 5 p. 100 (solution E).

b) Obtention du filtrat :

Cas des laits normaux ou formolés : Dans une fiole jaugée de $200 \mathrm{ml}$, mesurer exactement $40 \mathrm{ml}$ de lait bien homogénéisé. Dans le cas de laits altérés, une homogénéisation correcte est obtenue par l'appareil de L. Martelli et P. Navellier [3].

Ajouter :

- $50 \mathrm{ml}$ environ d'eau distillée; agiter ;

$-8 \mathrm{ml}$ de solution $\mathrm{A}$; agiter ;

$-8 \mathrm{ml}$ de solution $\mathrm{B}$; agiter; 
- 2 gouttes de solution $\mathrm{C}$; agiter ; ballon.

- 5 à $10 \mathrm{ml}$ d'eau distillée, à la pissette, en rinçant le col du

Neutraliser, en agitant, jusqu'à teinte légèrement rose, avec la solution D.

Compléter au trait de jauge et ajouter, à la pipette, $4 \mathrm{ml}$ d'eau distillée pour compenser le volume du coagulum. Mélanger soigneusement. Attendre 10 minutes environ. Filtrer sur filtre sec. Le filtrat doit être limpide. Il est parfois légèrement rose, mais cela est sans conséquence pour le dosage.

Cas des laits bichromatés : pour l'élimination nécessaire du bichromate, procéder comme suit :

Dans une fiole jaugée de $200 \mathrm{ml}$, mesurer exactement $40 \mathrm{ml}$ de lait bien homogénéisé.

Ajouter :

- $50 \mathrm{ml}$ environ d'eau distillée ; agiter ;

- $8 \mathrm{ml}$ de solution $\mathrm{A}$; agiter;

$-8 \mathrm{ml}$ de solution $\mathrm{B}$; agiter ;

- 2 gouttes de solution $\mathrm{C}$; agiter ; ballon.

- 5 à $10 \mathrm{ml}$ d'eau distillée à la pissette; en rinçant le col du

Attendre 2 minutes. Neutraliser, en agitant, à l'aide de la solution D. Après obtention d'une teinte rosée, ajouter un $\mathrm{ml}$ de solution D. Agiter. Le contenu de la fiole devient franchement rose. Rincer, si nécessaire, le col du ballon avec une petite quantité d'eau distillée.

Attendre 5 minutes environ.

Ajouter $20 \mathrm{ml}$ de solution $\mathrm{E}$; agiter.

Attendre de 10 à 15 minutes.

Ajuster au trait de jauge ; ajouter $4 \mathrm{ml}$ d'eau distillée supplémentaire et agiter. Laisser reposer quelques instants et filtrer sur filtre sec. Le filtrat est limpide, mais plus ou moins rose.

2) Solutions-étalons.

Solution-étalon I :

— Lactose hydraté : .............. 49,500 g

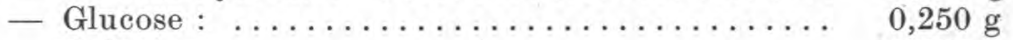

- Galactose : ................. 0,250 g

- Eau distillée : ............... q.s.p. $1000 \mathrm{ml}$

Cette solution correspond à une teneur en lactose hydrolysé de $0,5 \mathrm{~g} / \mathbf{1}$. 
Solution-étalon II ;

- Lactose hydraté : ................ 49,000 g

- Glucose : .................... 0,500 g

- Galactose : ................... 0,500 g

- Eau distillée : ................. q.s.p. $1000 \mathrm{ml}$

Cette solution correspond à une teneur en lactose hydrolysé de $1 \mathrm{~g} / 1$.

\section{B. - Chromatographie en couche mince.}

1) Matériel et réactifs.

- Equipement pour chromatographie en couche mince avec plaques de $200 \times 200 \mathrm{~mm}$ et de $200 \times 100 \mathrm{~mm}$, dispositif d'étalement, portoir pour plaques et enceinte de conservation, cuves de développement pour chaque format (1) ;

- Micropipettes de $10 \mathrm{~mm}^{3}$, graduées en $\mathrm{mm}^{3}$, à pointe fine;

- Etuve à $105^{\circ}$;

- Lampe à U. V., type Camag (2) ;

- Kieselgel G, Merck;

- Méthyléthylcétone pure Prolabo;

- Acide acétique $(\mathrm{d}=1,055-1,064)$;

- Méthanol RP;

- Aniline RP;

- Acide orthophtalique RP ;

- Butanol RP.

2) Préparation des plaques.

Le support chromatographique est constitué par un adsorbant solide mis en suspension, au moment de la préparation, dans une phase liquide appropriée. Nous avons fait un certain nombre d'essais pour sélectionner le support le plus favorable à la séparation recherchée.

Conformément à ce qui avait déjà été établi par Stahl [2], il s'est confirmé que le Kieselguhr donne un effet de traînée important, surtout pour les fortes concentrations en sucre qu'exige la méthode, de sorte que nous lui avons préféré le Kieselgel G qui permet de bien meilleures séparations.

Comme diluant, nous avons retenu l'eau distillée de préférence au tampon de Sörensen ( $\mathrm{pH} 6,8)$, qui ne sépare qu'imparfaitement

(1) Equipement Desaga, Hauptstrasse 60, Schilessfach 407, Heidelberg (Allemagne Fédérale). Distribué en France par RoucaIre, 21, rue des Favorites, Paris $15^{\mathrm{e}}$ ou

Equipement SHandon LTD, 6, Cromwell Place, London SW 7 (Angleterre) Distribué en France par Prolabo, 12, rue Pellé, Paris 11 e.

(2) Paris-Labo, 7, rue du Cardinal Lemoine, Paris $5^{\mathrm{e}}$. 
glucose et galactose, de même qu'à l'acide borique N/10, qui n'apporte pas d'amélioration notable aux résultats.

Pour préparer les plaques, on mélangera donc rapidement dans un mortier $30 \mathrm{~g}$ de Kieselgel $\mathrm{G}$ et $60 \mathrm{ml}$ d'eau distillée, jusqu'à obtention d'une suspension homogène qui sera étalée sur les plaques suivant la technique de Stahl [2]. Le séchage se fera à l'air libre pendant 3 ou 4 heures. Une activation à l'étuve n'apporte pas une meilleure séparation et s'avère donc inutile. Les plaques sont ensuite conservées à l'abri de la poussière et d'une humidité excessive.

\section{3) Dépôt des substances à chromatographier.}

Les dépôts sont à aligner à $15 \mathrm{~mm}$ du bord inférieur de la plaque, avec un espacement de $15 \mathrm{~mm}$. Les emplacements prévus sont marqués de façon presque imperceptible par une touche légère au crayon.

Ils se font à la micropipette, par petites fractions, en séchant chaque fois à l'aide d'un sèche-cheveux, de manière à obtenir des taches d'un diamètre très réduit.

On dépose ainsi successivement :

$-1 \mathrm{~mm}^{3}$ de la solution-étalon 1 (soit $0,250 \mu \mathrm{g}$ de glucose + $0,250 \mu \mathrm{g}$ de galactose $+49,5 \mu \mathrm{g}$ de lactose) ;

$-5 \mathrm{~mm}^{3}$ de filtrat lactique ;

- $1 \mathrm{~mm}^{3}$ de la solution-étalon II (soit $0,5 \mu \mathrm{g}$ de glucose $+0,5$ $\mu \mathrm{g}$ de galactose $+49 \mu \mathrm{g}$ de lactose),

de façon à encadrer chaque échantillon à analyser par 2 étalons.

\section{4) Développement.}

Il se fait dans une cuve de taille correspondant à celle de la plaque, en atmosphère saturée. La saturation est obtenue par l'application, sur les deux grandes faces de la cuve si elle est parallélépipèdique, ou sur sa totalité si elle est cylindrique, de feuilles d'un papier filtre de bonne qualité ou de papier ehromatographique, feuilles qui seront imprégnées de solvant.

Après différents essais portant sur la nature du solvant, nous avons retenu le mélange méthyléthylcétone/acide acétique/méthanol dans les proportions $60 / 20 / 20$, ce qui donne des résultats satisfaisants et permet la séparation glucose-galactose.

Les quantités à employer sont : $100 \mathrm{ml}$ pour les cuves parallélépipédiques, $70 \mathrm{ml}$ pour les cuves cylindriques de $12 \times 22 \mathrm{~mm}$.

La plaque, chargée de ses dépôts, est introduite verticalement dans la cuve saturée au préalable et close de façon étanche. L'ascension du solvant est arrêtée lorsque le front du liquide à atteint une ligne tracée antérieurement à $100 \mathrm{~mm}$ au-dessus des dépôts. Cette migration dure généralement de 20 à 25 minutes. 

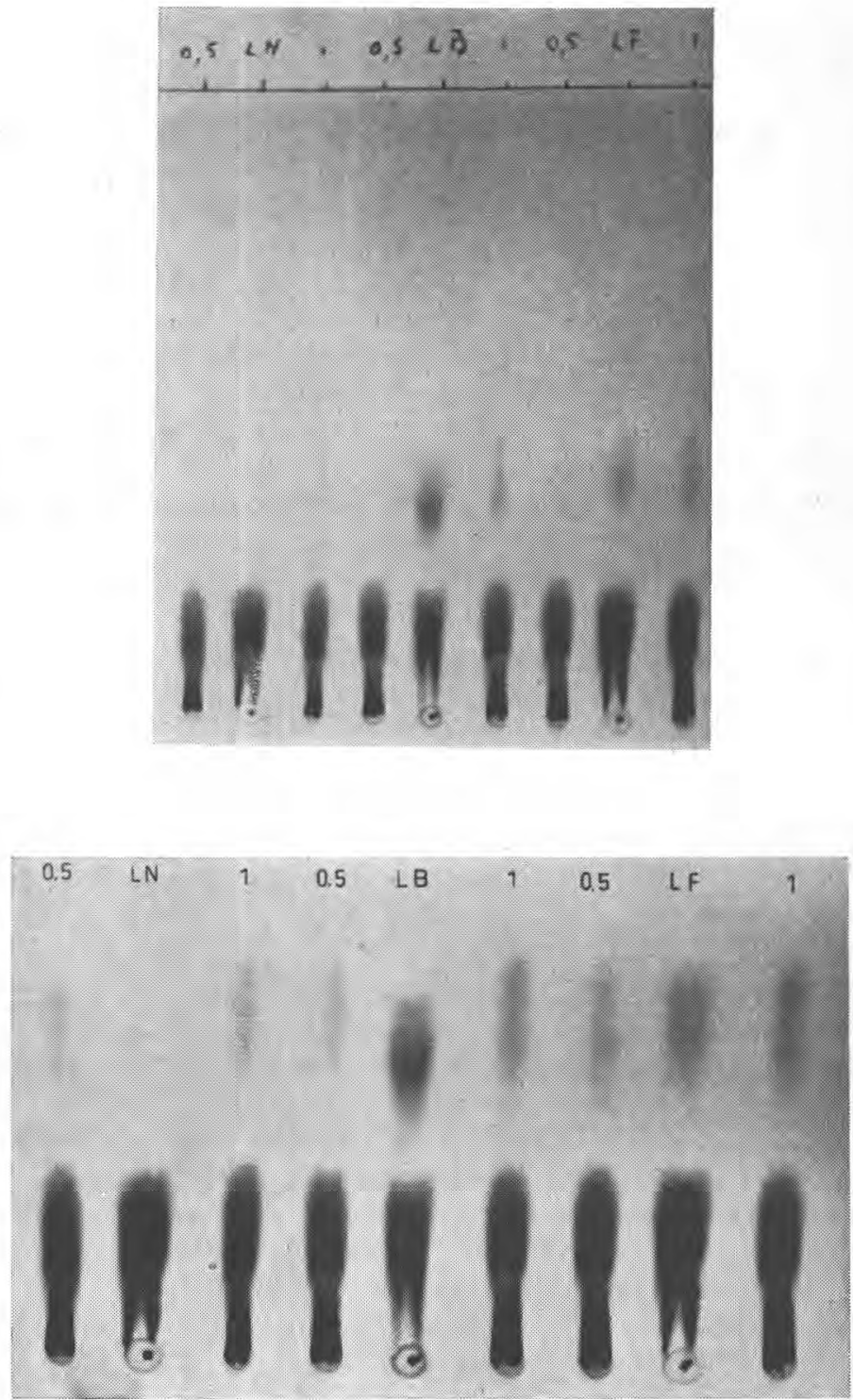

Fig. 1. - Appréciation de l'hydrolyse du lactose dans un lait normal (LN), un lait bichromaté (LB) un lait formolé (LF). En haut : lecture en lumière normale; en bas : lecture en lumière ultra-violette. 
5) Révélation.

Au terme de la migration la plaque est retirée de la cuve et placée horizontalement pour le séchage qui, le cas échéant, pourra être accéléré à l'aide du sèche-cheveux.

On pulvérise ensuite sur toute la zone intéressante, de façon uniforme, avec précaution et sans excès, la solution de phtalate d'aniline fraîchement préparée. Ce réactif s'obtient en dissolvant $0,93 \mathrm{~g}$ d'aniline récemment redistillée et $1,66 \mathrm{~g}$ d'acide orthophtalique dans $100 \mathrm{ml}$ de $\mathrm{n}$-butanol saturé d'eau.

Des essais comparatifs avec 5 autres révélateurs (o-aminophénol + acide phosphorique; acide 3,5 - dinitrobenzoïque ; acide 3,5 - dinitrosalicylique; chlorure de $2,3,5$ - triphényltétrazole; anisaldéhyde) nous ont fait retenir le phtalate d'aniline comme étant le plus sensible.

Après aspersion, les plaques séjournent 15 minutes à l'étuve à $105^{\circ}$. Les glucides séparés apparaissent sous forme de taches brunes qui se conservent bien et même se renforcent à l'obscurité.

6) Lecture et interprétation.

Elle peut se faire en lumière normale. Le lactose forme une tache considérable, allongée, dont l'effet de traînée, dû à la quantité massive déposée, s'étend souvent jusqu'au dépôt initial. Galactose et glucose, qui'ont un Rf supérieur, se situent plus haut et forment deux taches d'intensité beaucoup plus faible, voisines, dont la séparation est quelquefois imparfaite, mais toujours suffisante pour l'identification des deux sucres.

Les taches faibles deviennent beaucoup plus visibles en lumière ultra-violette de $350 \mathrm{~m} \mu$, ce qui facilite notablement la lecture. Des taches de $0,03 \mu \mathrm{g}$ peuvent encore être décelées dans ces conditions.

L'interprétation consiste à comparer l'intensité de la double tache du galactose-glucose du filtrat à celles des doubles taches étalons qui l'encadrent. Si l'intensité fournie par le filtrat est inférieure à celle de l'étalon $\mathrm{I}$, on peut en conclure que le lait correspondant contenait moins de $0,5 \mathrm{~g} / 1$ de lactose hydrolysé et que, pour un dosage réductrimétrique exact du diholoside, une hydrolyse totale n'est pas nécessaire, alors qu'elle s'imposerait dans le cas contraire. Le rôle de la solution-étalon II est d'abord de permettre une meilleure localisation des deux hexoses du filtrat lorsqu'ils sont seulement présents à l'état de traces et ensuite de faciliter l'appréciation semi-quantitative de ces deux taches par comparaison avec deux intensités nettement différentes, donc la classification dans l'une des trois catégories :

- Lactose hydrolysé inférieur à $0,5 \mathrm{~g} / 1$;

- Lactose hydrolysé compris entre $0,5 \mathrm{~g} / 1$ et $1 \mathrm{~g} / 1$;

- Lactose hydrolysé supérieur à 1 g/1. 
La figure 1 présente un exemple d'application de la technique. Elle concerne l'analyse des filtrats de 3 laits différents désignés par :

LN : lait de consommation pasteurisé, acheté en bouteille capsulée, datant de deux à trois jours ;

LB : lait cru bichromaté $(0,250 \mathrm{~g}$ de bichromate pour $250 \mathrm{ml})$, âgé de 4 mois $1 / 2$. L'antiseptique a été ajouté environ 3 heures après la traite;

LF : lait cru formolé ( 2 gouttes de formol $+0,5 \mathrm{~g}$ de trioxyméthylène pour $250 \mathrm{ml}$ ).

Les laits LB et LF présentaient les caractères de laits fortement altérés; en particulier, le lait bichromaté avait acquis, au cours du stockage, une teinte verdâtre caractéristique de la réduction du bichromate.

Chaque filtrat se trouve encadré par les deux solutions-étalons. Malgré l'imperfection du cliché, on peut conclure, surtout en lumière ultra-violette :

- que le lactose du lait normal était hydrolysé à un taux inférieur à $0,5 \mathrm{~g} / 1$;

- que le lactose des laits bichromaté et formolé était hydrolysé à un taux supérieur à $1 \mathrm{~g} / 1$.

\section{III. - Discussion et conclusion}

De même qu'en chromatographie sur papier, la détection semi-quantitative des sucres par la technique en couche mince n'est à entreprendre que lorsque la fermentation lactique a été seule à se développer dans le lait. Dans l'éventualité d'autres fermentations, facilement décelables, il est préférable de mettre en œuvre d'autres méthodes ou encore, en cas d'altérations profondes, de ne pas chercher à définir le lait par une teneur en lactose qui serait sans signification, mais par le dosage d'autres éléments moins dégradés. C'est là une limite de la technique, mais elle ne lui est pas spécifique.

Dans tous les cas où elle est applicable, la méthode préconisée présente, par rapport à celle sur papier, d'indiscutables avantages.

Elle ne nécessite qu'un matériel de faible encombrement et relativement peu couteux. En particulier, elle ne demande pas une enceinte thermostatée comme l'exige presque toujours la chromatographie sur papier.

La consommation en réactifs est très faible.

Les manipulations préparatoires sont, en outre, notablement simplifiées. Du fait que le support mince de gel de silice tolère la présence d'électrolytes dans une mesure beaucoup plus large 
que le papier, il n'est plus nécessaire de procéder à une défécation acétique suivie d'une reconcentration du produit dilué, ce qui représente un gain de temps important. Dans notre méthode, la dilution par 5 introduite par la défécation au ferrocyanure de $\mathrm{K}$ ou au chlorure de Ba est compensée de façon simple par un dépôt cinq fois supérieur sur la plaque.

Enfin, alors que sur papier la durée totale des opérations est d'une quarantaine d'heures en employant du papier Whatmann no 4 ou même de 80 heures avec un papier plus lent (Arches 302), celle de l'ensemble des opérations en couche mince peut être achevée en 2 heures. Comme chaque plaque de $200+200 \mathrm{~mm}$ peut facilement recevoir 12 dépôts et que deux plaques peuvent être développées ensemble dans la même cuve, 8 laits pourront donc être analysés rapidement, en une seule opération, pour un prix de revient très modique. De la sorte on saura, dans un délai très court, si le dosage du lactose dans un échantillon de lait peut être fait avec ou sans hydrolyse totale, la détermination de la teneur en chlorure pouvant, par ailleurs, être réalisée sur le même lactosérum.

Pour toutes ces raisons, il nous semble que la chromatographie en couche mince devrait se substituer peu à peu à la chromatographie sur papier dans ce domaine de l'analyse alimentaire.

\section{Summary}

Before performing a "reductimétric " determination of lactose in milk, it is essential to ascertain whether there was no more than $0,5 \mathrm{~g}$ of broken down lactose by litre.

That verification, which is till now carried out in about 40 hours by paper chromatography, can be completed in 2 hours, in the easier and the more inexpensive way, thanks to chromatography of silicagel in thin layer, whose process is described and discussed.

\section{RÉFERNCES BIBLIOGRAPHIQUES}

[1] Mme Saenz Lascano Ruiz I. (1954). - Le dosage du lactose dans les laits altérés par fermentation lactique. Les conditions d'hydrolyse quantitative du lactose dans un lactosérum. Ann. Fals., 47, pp. 167 174 et 285-305.

[2] Stahl E. (1962). - Dünnschicht-Chromatographie pp. 473-481. Springer-Verlag Berlin.

[3] Mme Martelli L., Navellier P. (1952). - Homogénéisation mécanique des échantillons de laits frais ou altérés. Ann. Fals., 45, no 519 520, pp. 101-108.

(Ecole de Laiterie de Naney, Directeur : A. VEILLET). 